\title{
Campylobacter jejuni-associated perimyocarditis: two case reports and review of the literature
}

\author{
Fredrik Hessulf ${ }^{1 *}$, Johan Ljungberg ${ }^{2}$, Per-Anders Johansson², Mats Lindgren² and Johan Engdahl ${ }^{2,3}$
}

\begin{abstract}
Background: Campylobacter spp. are among the most common bacterial causes of gastroenteritis world-wide and mostly follow a benign course. We report two cases of Campylobacter jejuni-associated perimyocarditis, the first two simultaneous cases published to date and the third and fourth cases over all in Sweden, and a review of the literature.

Case presentation: A previously healthy 24-yo male (A) presented at the Emergency Department(ED) with recent onset of chest pain and a 3-day history of abdominal pain, fever and diarrhoea. The symptoms began within a few hours of returning from a tourist visit to a central European capital. Vital signs were stable, the Electrocardiogram(ECG) showed generalized ST-elevation, laboratory testing showed increased levels of C-reactive protein(CRP) and high-sensitive Troponin T(hsTnT). Transthoracic echocardiogram (TTE) was normal, stool cultures were positive for $C$ Jejuni and blood cultures were negative. Two days after patient A was admitted to the ED his travel companion (B), also a previously healthy male (23-yo), presented at the same ED with almost identical symptoms: chest pain precipitated by a few days of abdominal pain, fever and diarrhoea. Patient B declared that he and patient A had ingested chicken prior to returning from their tourist trip. Laboratory tests showed elevated CRP and hsTnT but the ECG and TTE were normal. In both cases, the diagnosis of $C$ jejuni-associated perimyocarditis was set based on the typical presentation and positive stool cultures with identical strains. Both patients were given antibiotics, rapidly improved and were fully recovered at 6-week follow up.
\end{abstract}

Conclusion: Perimyocarditis is a rare complication of $C$ jejuni infections but should not be overlooked considering the risk of heart failure. With treatment, the prognosis of full recovery is good but several questions remain to be answered regarding the pathophysiology and the male preponderance of the condition.

Keywords: Campylobacter jejuni, Perimyocarditis, High-sensitive Troponin T, ST-elevation

\section{Background}

Campylobacter spp. are among the most common bacterial causes of gastroenteritis world-wide and usually the result of handling and consumption of poultry meat [1]. Typical symptoms include diarrhoea, fever and abdominal cramps. Infections are sometimes asymptomatic, often self-limiting and rarely require antibiotics. Bacteraemia is seen in less than $1 \%$ of cases, acute and post-infectious complications are rare but include arthritis, meningitis, endocarditis, sepsis and Guillain-Barré syndrome $[2,3]$. Myocarditis and perimyocarditis are

\footnotetext{
* Correspondence: fredrik.hessulf@regionhalland.se

${ }^{1}$ Department of Anaesthesiology and Intensive Care Medicine, Hallands

Hospital, Halmstad, Sweden

Full list of author information is available at the end of the article
}

known but rare complications of $C$ jejuni infections. Here we present an unusual case where two previously healthy males presented at the ED with diarrhoea and chest pain and where the diagnosis of $C$ jejuni-associated perimyocarditis was set. We also present the results of a review of the literature and discuss disease characteristics i.e., the preponderance of young healthy males and pathophysiological mechanisms.

\section{Case presentation}

\section{Case 1}

A previously healthy 24-year-old male (A) presented at the emergency department (ED) with chest pain and diarrhoea. The chest pain began $2 \mathrm{~h}$ prior to hospital admission and was described as a constant light chest 
pressure without correlation to breathing or body position. 3 days earlier, the patient and a friend had had chicken in a restaurant before boarding a flight to Sweden. One hour after arrival in Sweden the patient experienced sudden abdominal pain, chills and diarrhoea. During the following three days the patient had 6 diarrhoeas per day, mucous but no visible blood. The patient was diagnosed with $C$ jejuni gastroenteritis after stool culture at a primary care facility, no antibiotics were given. Upon admission to the ED the abdominal pain had subsided, the patient was afebrile $\left(37^{\circ} \mathrm{C}\right.$ or $\left.98.6{ }^{\circ} \mathrm{F}\right)$ but still experiencing diarrhoea. Upon examination the patient had normal circulatory and respiratory parameters. Heart auscultation: regular rhythm (RR), no murmurs or extra sounds. Lung and abdominal examinations were normal. Laboratory examinations showed C-reactive protein $(\mathrm{CRP})$ at 89.1 (normal range $<10 \mathrm{mg} / \mathrm{L}$ ), leukocyte count (LC) at 11.3 (normal range $4-12 \times 10^{9} / \mathrm{L}$ ) and highsensitive Troponin $\mathrm{T}$ (hsTnT) at 108 (normal range $<14 \mathrm{ng} / \mathrm{L}$ ) ECG showed regular sinus rhythm, 64/min and general $1 \mathrm{~mm}$ ST-elevation (Fig. 1). The patient was treated with Brufen $200 \mathrm{mg}$ (tid), Omeprazol $20 \mathrm{mg}$ (qd) and Loperamid $2 \mathrm{mg}$ and admitted to a cardiac care unit (CCU) for cardiac monitoring.

During the following 4 days the hsTnT reached a maximum value of 504 and then dropped to 46 . Stool culture confirmed the diagnosis of $C$ jejuni. Blood cultures were negative. Transthoracic echocardiogram (TTE) showed normal right and left ventricle function, ejection fraction (EF) 60-65\%, normal valvular structure and function, no hypokinesia or pericardial effusion. After on day the ECGchanges had resolved. The patient was started on ciprofloxacin but developed urticarial rashes and severe itching and the treatment was discontinued. The chest pain subsided after 2 days and the patient left the hospital after 4 days. The patient was given a 10 day prescription of Azithromycin $500 \mathrm{mg}$ (qd) upon hospital release. The diagnosis was determined to be $C$ jejuni-associated perimyocarditis (CPM). At follow-up visit 4 weeks after discharge the patient was without complaints, physical examination was normal and both ECG and TTE were normal.

\section{Case 2}

Two days after A was admitted to the ED with chest pain, his travelling companion (B) presented on the same ED with a 48-h history of mild constant chest pain exacerbated by breathing but not body position. B had, in the same fashion as his companion A, developed abdominal pain, diarrhoea and chills upon return to Sweden. After initially experiencing multiple diarrhoeas daily, upon admission to the ED the diarrhoeas had started to subside and the abdominal pain resolved. The patient was afebrile $\left(37.4^{\circ} \mathrm{C}\right.$ or $\left.99.3^{\circ} \mathrm{F}\right)$, circulatory and respiratory parameters as well as heart, lung and abdominal examination were normal. Laboratory tests showed CRP at 46.5, LPK at 9.1 and hsTnT at 128. The ECG was normal. The patient was admitted to a CCU for hsTnT-serial testing and cardiac monitoring.

During the hospital stay the hsTnT reached a maximum of 128 before dropping to 52 . Stool cultures confirmed the

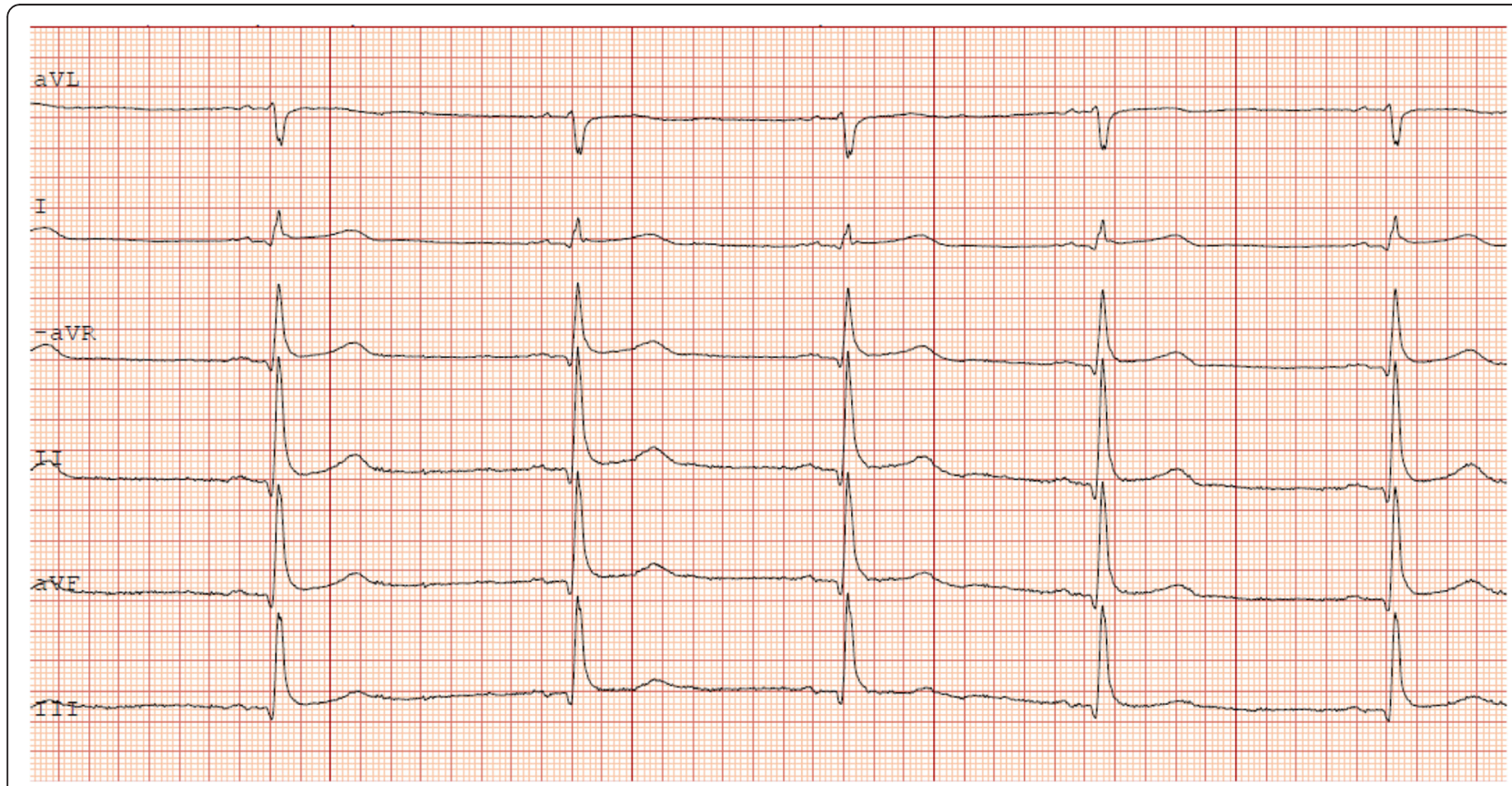

Fig. 1 Limb leads from 12-lead ECG from patient A showing typical generalized ST-segment elevation 
diagnosis of $C$ jejuni sensitive to Azithromycin. Blood cultures were negative. TTE showed normal right and left ventricle function, normal valvular structure and function and no pericardial effusion. The chest pain resolved within $48 \mathrm{~h}$ and the patient was released from the hospital with a 10-day prescription of Azithromycin $500 \mathrm{mg}$ qd. The patient was given the diagnosis of $C$ jejuni-associated perimyocarditis (CPM). At follow-up visit 4-6 weeks after discharge the patient was doing well, physical examination, stress ECG and TTE were all normal.

\section{Discussion}

$C$ jejuni infections are common but rarely lead to severe symptoms outside of the gastrointestinal tract [2]. To date only a few case reports $[4,5]$ have described $C$ jejuniassociated myocarditis/perimyocarditis (CPM) despite the fact that $C$ jejuni infections are one of the most common causes of gastroenteritis word wide. In this case report we present an unusual case of two previously healthy young males who, after eating chicken at a restaurant, develop typical gastroenteritis symptoms (abdominal pain, diarrhoea, chills) within $24 \mathrm{~h}$ and within $48-72 \mathrm{~h}$ of the onset of gastroenteritis symptoms also develop chest pain. Based on elevated hsTnT, typical ECG-findings (case A), positive stool cultures, a lack of alternative plausible explanations and a temporal association, the diagnosis of $C$ jejuni-associated perimyocarditis was set. Subsequent analysis of both stool cultures demonstrated the presence of the same $C$ jejuni strains with identical genotypes. At follow up 4-6 weeks after discharge the patient in case one was asymptomatic, the patient in case 2 complained of unspecific fatigue that had resided 3 weeks later. Both patients had normal TTE and stress ECG examinations.

This case report highlights several points of interest regarding pathogenesis and epidemiology of campylobacterassociated perimyocarditis. Here follows a review of the literature and further discussion of the case report.

A search on MEDLINE/PubMed using any combination of the MESH-terms "Campylobacter", "Campylobacter jejuni" "myocarditis", "perimyocarditis" and "pericarditis" yielded 34 relevant articles 1979-2015. After cross checking the reference lists two additional articles were found. Including the characteristics of the two case reports presented above(Case A and B), a total of 44 cases; 20 cases of myocarditis, 19 cases of perimyocarditis and five cases of pericarditis associated with $C$ jejuni infection have been described in the literature. We review the baseline characteristics of the patients based on the published case reports (Table 1), and summarize the most recent case reports in more detail (Table 2), review the clinical presentation of $C$ jejuni myocarditis, differential diagnosis and diagnostic issues, treatment, pathophysiology and certain disease characteristics.
Table 1 Summary of baseline characteristics of all published case reports including patient $A$ and patient B. $n=44$ $[4-6,10-12,14,18,22-49]^{a, b}$

\begin{tabular}{lll}
\hline Characteristics & & \\
\hline Age (years) & Mean & 29,4 \\
& Max & 60 \\
Myocarditis $n$ (\%) & All & 15 \\
& Female & $20(100)$ \\
Perimyocarditis $n$ (\%) & Male & $0(0)$ \\
& All & $20(100)$ \\
Pericarditis $n(\%)$ & Female & $19(100)$ \\
& Male & $1(5)$ \\
& All & $18(95)$ \\
& Female & $4(100)$ \\
& Male & $3(75)$ \\
\end{tabular}

${ }^{a}$ Campylobacter not identified to the species level (ref [26] and [28])

${ }^{b}$ Mean age (43 years old) of the patient series which included one case of Campylobacter (ref [28])

The mean age was 29 years, $93 \%$ of cases affected men. Few patients had multiple comorbidities, a majority of patients were previously healthy, but there were also a substantial amount of cases that affected immunocompromised patients.

The clinical presentation is variable and there are no pathognomonic symptoms or signs. Typically, the patient presents with a history of a few days of gastroenteritis (fever, abdominal pain, diarrhoea) and recent onset of chest pain/discomfort/tightness. Vital signs are often in the normal range. The ECG often shows signs of tachycardia and generalized ST-elevation/depression/Twave inversion, easily mistaken for an acute coronary syndrome resulting in normal coronary angiography examinations. Additional differential diagnosis includes valvular pathology and pulmonary embolism. Laboratory investigations generally show elevated levels of cardiac enzymes (Troponin T/I or Creatinine Kinases MB Isoenzyme (CKMB) as well as inflammation markers (CRP). Additional diagnostic measures have traditionally included TTE and, more recently, cardiac Magnetic Resonance Imaging (MRI). The TTE can sometimes reveal decreased left ventricular ejection fraction (LVEF), wall motion abnormalities and pericardial effusion. Cardiac MRI is a non-invasive method that has gained popularity lately. Subepicardial and myocardial enhancement can be seen primarily in the left ventricle. Based on the combination of symptoms (abdominal and chest symptoms) and signs (ECG-changes and laboratory investigations) the diagnosis of myocarditis is suspected and the patient admitted to a cardiac care unit (CCU) for continuous cardiac monitoring. The cardiac monitoring is often uneventful but at least on case of severe arrhythmia 
Table 2 Summary of the most recent case reports including case 1 (patient $A)^{a}$ and 2 (patient $\left.B\right)^{b} \cdot n=14$

\begin{tabular}{|c|c|c|c|c|c|c|}
\hline Sex/Age & Cardiac marker & ECG & Echo & MRI & Antibiotics & Outcome \\
\hline $\bar{M} / 24^{a}$ & TnT 504 ng/L & ST-elevation & Normal, LVEF 60-65 \% & No & Azithromycin & Full recovery \\
\hline $\mathrm{M} / 23^{\mathrm{b}}$ & TnT 128 ng/L & Normal & Normal incl LVEF & No & Azithromycin & Full recovery \\
\hline$M / 60[6]$ & N/A & N/A & Pericard effusion & No & $\begin{array}{l}\text { Ceftriaxone/ } \\
\text { Meropenem }\end{array}$ & Diarroea \\
\hline M/43 [7] & $\mathrm{TnT} 1.75 \mathrm{ng} / \mathrm{ml}$ & ST-elevation & $\begin{array}{l}\text { Abn wall motion } \\
\text { LVEF } 68 \%\end{array}$ & $\begin{array}{l}\text { Subepi/myocardial } \\
\text { enhancement }\end{array}$ & Azithromycin & N/A \\
\hline M/33 [8] & Tnl $18.6 \mathrm{mcg} / \mathrm{L}$ & ST-elevation & LVEF $56 \%$ & $\begin{array}{l}\text { Increased signal subepi, } \\
\text { dilated LV/RV }\end{array}$ & Roxithromycin & N/A \\
\hline $\mathrm{M} / 17[9]$ & Tnl 16.8 ng/mL & ST-elevation & Normal & Subepicardial enhancement & Azithromycin & Full recovery \\
\hline $\mathrm{M} / 21[5]$ & Tnl $15.6 \mathrm{ng} / \mathrm{ml}$ & Normal & Decreased LVEF & $\begin{array}{l}\text { Subepi/myocardial } \\
\text { enhancement }\end{array}$ & Azithromycin & Almost full rec \\
\hline M/24 [5] & Tnl $8.9 \mathrm{mcg} / \mathrm{L}$ & ST-elevation & LVEF $40 \%$ & Subepicardial enhancement & No & Full recovery \\
\hline M/42 [5] & $\mathrm{Tnl} 11.6 \mathrm{mcg} / \mathrm{L}$ & ST-elevation & LVEF $40 \%$ & Subepicardial enhancement & Ciprofloxacin & Full recovery \\
\hline $\mathrm{M} / 21[10]$ & Tnl 39.8mcg/L & ST-elevation & Normal inkc LVEF & No & Ciprofloxacin & Full recovery \\
\hline $\mathrm{M} / 21[11]$ & $\mathrm{Tnl} 2.5 \mathrm{mcg} / \mathrm{L}$ & ST-elevation & Normal incl LVEF & No & Yes(unknown kind) & Full recovery \\
\hline $\mathrm{M} / 24$ [12] & CK normal & Arrythmia & $\begin{array}{l}\text { Decreased LVEF/ } \\
\text { peric effusion }\end{array}$ & No & Roxithromycin & N/A \\
\hline $\mathrm{M} / 19$ [13] & $\mathrm{TnT} 0.52 \mathrm{ng} / \mathrm{mL}$ & Strain & N/A & Yes & N/A & N/A \\
\hline$M / 16[14]$ & $\mathrm{TnT} 1.7 \mathrm{ng} / \mathrm{mL}$ & ST-elevation & LVEF $45 \%$ & No & Clarythromycin & Full recovery \\
\hline$M / 17[14]$ & $\operatorname{TnT} 0.9 \mathrm{ng} / \mathrm{mL}$ & ST-elevation & Normal incl LVEF & Myocardial enhancement & Clarythromycin & Full recovery \\
\hline
\end{tabular}

(ventricular tachycardia) has been described [6]. Historically, endomyocardial biopsy (EMB) has been performed and is considered the gold standard for diagnosing myocarditis. However, there is a risk of complications related to the procedure (although probably less common than previously thought [7]. According to the AHA/ACCF/ESC joint statement [8] and a recent review [9] EBM should be performed in patients with severe symptoms (heart failure, dilated cardiomyopathy, hemodynamic compromise) where the risks are outweighed by the potential benefits. Pena et al. [10] reported a rare case of $C$ jejuni-associated myocarditis with pathological evidence of inflammation (but no evidence of $C$ jejuni on polymerase chain reaction (PCR) study of the myocardium).

Treatment can be divided into supportive and causal. Supportive treatment consists of preventing dehydration and electrolyte disturbances and managing eventual heart failure (Angiotensin receptor blocker/B adrenergic blocker) or arrhythmias. Causal treatment consists of antibiotic treatment; primarily Macrolides (Azithromycin/Roxithromycin/Clarythromycin) and Flouroquinolones (Ciprofloxacin) have been used. To the best of our knowledge there is no consensus on dosage and treatment duration; when reviewing recent cases the most common choice of antibiotic was the Macrolide Azithromycin, $500 \mathrm{mg}$ qd for 310 days [11] Full recovery and return to normal physical status was noted in a vast majority of published cases. Complications include persistent heart failure [12] and ventricular arrhythmia [6]. Few cases with severe and fatal outcome have been described in the literature, and all but one had pre-existing conditions [10].

This case report highlights 3 points of interest.

1) We are, to our knowledge, the first to type a $C$ jejuni strain associated with myocarditis. Using Pulse-field gel electrophoresis (PFGE) and the restriction enzyme SMAL the two campylobacter isolates showed identical patterns (Fig. 2) and the same pattern of antibiotic resistance. No match of a similar strain could be found in the Swedish database of $C$ jejuni strains, and the only similar strain we could find was from a Swiss chicken isolate from 2008 and this isolate was not associated with any disease. The two cases in this report are also the first ever described where the same $C$ jejuni strain caused myocarditis in two related cases. All previous case reports have reported single cases of myocarditis. This raises the question whether different campylobacter strains have different affinity for the myocardium. Our two cases represent the third and fourth case of myocarditis ever described in Sweden, which highlights the rare nature of the condition.

2) Our case report also highlight the need for further investigation of the mechanisms of campylobacterassociated myocarditis. Earlier studies have speculated that there might be at least two types of CPM: one bacteria/toxin mediated that causes acute symptoms within 2-4 days of the gastroenteritis and 


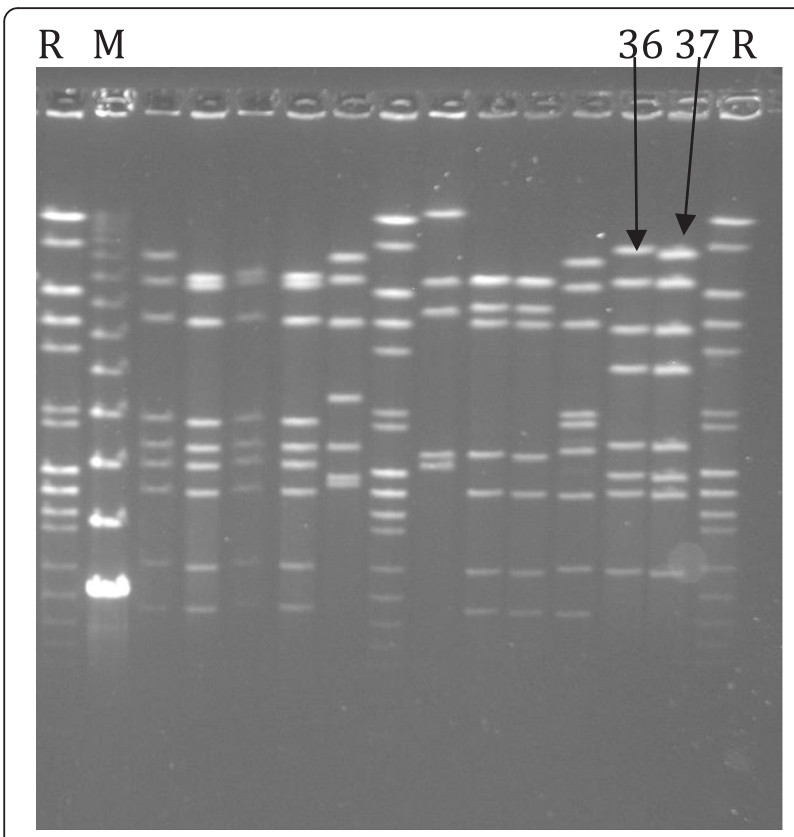

Fig. 2 Pulsed-field gel electrophoresis according to the standardized Campynet protocol and using Smal restriction enzyme. R: normalisation standard; M: molecular size marker; 36 and 37 show the banding patterns of the $C$. jejuni isolates from patient $A$ and $B$, respectively

another, immunological type, that causes delayed symptoms 2 weeks after the onset of the infection [13]. Neither type has been particularly well characterized, there is to our knowledge only one case of $C$ jejuni-myocarditis with pathological confirmation [10]. Alzand et al. speculate that several mechanisms, apart from direct bacterial invasion, such as bacterial toxins, circulating immune complexes or cytotoxic T-cells might be involved [14]. In 2007 Becker et at studied a Danish cohort of 6204 cases of $C$ jejuni-gastroenteritis and found no increase in myocarditis incidence in the $C$ jejuni cohort and no cases of pericarditis as compared to age matched controls. Because of the patient selection, retrospective study design and the very low incidence of myocarditis (1/6204) and pericarditis (0/6204) in the $C$ jejuni-cohort the authors limited their conclusion to state that they did not observe an increased incidence of myocarditis or pericarditis. The authors offered an intriguing but speculative hypothesis for the aetiology of campylobacter-associated myocarditis: the possibility of a viral co-infection that might be the actual cause of the perimyocarditis/myocarditis.

Because of the relatively acute onset of chest pain in relation to the debut of the infection, one might speculate that the most probable cause of myocarditis in this case report is a bacteria/toxin effect. The fact that blood cultures were negative does not rule out transient bacteraemia or a toxin effect. Less than 1 out of 100 Campylobacter gastroenteritis infections have positive blood cultures [15] which have led some to believe that we under-diagnose Campylobacterimia [16]. A recent study by Harvala et al. showed an increase in the number of Campylobacteremia cases in Sweden in 2014 as a result of improvements in blood culture medium [17] This fact could explain why, in the presently described as well as in all previously described cases of myocarditis/perimyocarditis/ pericarditis apart from one case of pericarditis [18], blood cultures have been negative. This patient was also immunocompromised due to an X-linked Agammaglobulinemia, and one can speculate that this could affect the disease mechanism.

3) This case report and literature review also highlights the fact that (young) men seem to be affected by campylobacter-associated myocarditis/perimyocarditis to a larger extent than women. The incidence of $C$ jejuni gastroenteritis is slightly higher for men than women but almost all reported CPM have affected men [2]. This observation has been made several times before, but no explanation has been offered. If one combines all reported cases of myocarditis and perimyocarditis associated with $C$ jejuni infection described in the literature to date, only $7 \%$ affect women (4/43). A comparison with the incidence of other bacteria-associated types of myocarditis is difficult because of lack of reliable studies. It is a wellknown fact that most types of cardiovascular disease have a higher incidence and prevalence among men than women (at least up to the age of 75) [19], and recent reports [20] have shown and confirmed a higher incidence of myocarditis in men as compared to women. The exact mechanisms for this is not known but some authors speculate that factors such as sex hormones (both testosterone as well as the female sex hormones), the immune system and genomics as well as differences in clinical manifestation and treatment might play a role [21].

\section{Conclusion}

Myocarditis and perimyocarditis are rare complications of $C$ jejuni infections that predominantly affect young previously healthy men and in the majority of cases follow a benign course. More studies are needed to elucidate the characteristics and pathogenesis of this rare condition.

\section{Abbreviations}

CKMB, creatinine kinase MB isoenzyme; CPM/CM/CP, campylobacter jejuniassociated perimyocarditis/myocarditis/pericarditis; CRP, C-reactive protein; ECG, electrocardiogram; ED, emergency department; EMB, endo-myocardial biopsy; hsTnT, high sensitive troponin; LC, leukocyte count; LVEF, left ventricle 
ejection fraction; MRI, magnetic resonance imaging; PCR, polymerase chain reaction; PFGE, pulse-field gel electrophoresis; T C jejuni, campylobacter jejuni; TTE, transthoracic echocardiogram

\section{Acknowledgements}

We thank associate professor Eva Olsson Engvall, National Veterinary Institute, Uppsala, Sweden for genotyping the C. jejuni isolates and for valuable comments to the manuscript.

\section{Funding}

No funding was obtained for this study.

\section{Availability of data and materials}

All data is contained in the manuscript.

\section{Authors' contributions}

$\mathrm{FH}$ drafted the manuscript, JE and FH revised the manuscript. JL was responsible for the microbiological analysis. FH, PA-J, ML and JE were involved in the care of the patient. All authors made important contributions and have read and approved the final version of the manuscript.

\section{Competing interests}

The authors declare that they have no competing interests.

\section{Consent for publication}

Written informed consent was obtained from the patients for publication of this Case report and any accompanying images. A copy of the written consent is available for review by the Editor of this journal.

\section{Ethics approval and consent to participate}

Not applicable.

\section{Author details \\ 'Department of Anaesthesiology and Intensive Care Medicine, Hallands Hospital, Halmstad, Sweden. ${ }^{2}$ Department of Internal Medicine, Hallands Hospital, Halmstad, Sweden. ${ }^{3}$ Department of Molecular and Clinical Medicine/Cardiology, Sahlgrenska Academy, University of Gothenburg, Gothenburg, Sweden.}

\section{Received: 24 December 2015 Accepted: 7 June 2016}

Published online: 14 June 2016

\section{References}

1. EFSA. Panel on Biological Hazards (BIOHAZ): Scientific Opinion on Campylobacter in broiler meat production: control options and performance objectives and/or targets at different stages of the food chain. EFSA J. 2011:9(4):2105-246.

2. Allos BM. Campylobacter jejuni Infections: update on emerging issues and trends. Clin Infect Dis. 2001;32(8):1201-6.

3. Kaakoush $\mathrm{NO}$ et al. Global epidemiology of Campylobacter infection. Clin Microbiol Rev. 2015;28(3):687-720

4. Cunningham $\mathrm{C}$, Lee $\mathrm{CH}$. Myocarditis related to Campylobacter jejuni infection: a case report. BMC Infect Dis. 2003:3:16.

5. De Cock D et al. Myocarditis associated with campylobacter enteritis: report of three cases. Circ Heart Fail. 2012;5(2):e19-21.

6. Nevzorov R et al. Campylobacter-associated myopericarditis with ventricular arrhythmia in a young hypothyroid patient. Isr Med Assoc J. 2010;12(8):505-6.

7. Holzmann $\mathrm{M}$ et al. Complication rate of right ventricular endomyocardia biopsy via the femoral approach: a retrospective and prospective study analyzing 3048 diagnostic procedures over an 11-year period. Circulation. 2008;118(17):1722-8.

8. Dickstein $\mathrm{K}$ et al. ESC Guidelines for the diagnosis and treatment of acute and chronic heart failure 2008: the Task Force for the Diagnosis and Treatment of Acute and Chronic Heart Failure 2008 of the European Society of Cardiology. Developed in collaboration with the Heart Failure Association of the ESC (HFA) and endorsed by the European Society of Intensive Care Medicine (ESICM). Eur Heart J. 2008:29(19):2388-442.

9. Sagar S, Liu PP, Cooper Jr LT. Myocarditis. Lancet. 2012;379(9817):738-47

10. Pena LA, Fishbein MC. Fatal myocarditis related to Campylobacter jejuni infection: a case report. Cardiovasc Pathol. 2007;16(2):119-21.
11. Fica A et al. A case of myopericarditis associated to Campylobacter jejuni infection in the southern hemisphere. Braz J Infect Dis. 2012:16(3):294-6.

12. Cox ID, Fluck DS, Joy MD. Campylobacter myocarditis; loose bowels and a baggy heart. Eur J Heart Fail. 2001;3(1):105-7.

13. Uzoigwe C. Campylobacter infections of the pericardium and myocardium. Clin Microbiol Infect. 2005;11(4):253-5.

14. Alzand BS et al. Campylobacter jejuni: enterocolitis and myopericarditis. Int J Cardiol. 2010;144(1):e14-6.

15. Skirrow MB et al. Campylobacter bacteraemia in England and Wales, 1981-91. Epidemiol Infect. 1993;110(3):567-73.

16. Louwen $\mathrm{R}$ et al. Campylobacter bacteremia: a rare and under-reported event? Eur J Microbiol Immunol (Bp). 2012;2(1):76-87.

17. Harvala $\mathrm{H}$, et al. Increased number of Campylobacter bacteremia cases in Sweden, 2014. Clin Microbiol Infect. 2016 Apr;22(4):391-3.

18. Rafi A, Matz J. An unusual case of Campylobacter jejuni pericarditis in a patient with X-linked agammaglobulinemia. Ann Allergy Asthma Immunol. 2002;89(4):362-7.

19. Franklin BA, Cushman M. Recent advances in preventive cardiology and lifestyle medicine: a themed series. Circulation. 2011;123(20):2274-83.

20. Magnani JW et al. Survival in biopsy-proven myocarditis: a long-term retrospective analysis of the histopathologic, clinical, and hemodynamic predictors. Am Heart J. 2006;151(2):463-70.

21. Fairweather D, Cooper Jr LT, Blauwet LA. Sex and gender differences in myocarditis and dilated cardiomyopathy. Curr Probl Cardiol. 2013;38(1):7-46.

22. Fradejas I, et al. Campylobacter jejuni pericarditis in a renal transplant recipient on sirolimus therapy. Clin Microbiol Newsl. 2015; 37(21)173-75.

23. Panikkath $\mathrm{R}$ et al. Chest pain and diarrhea: a case of Campylobacter jejuniassociated myocarditis. J Emerg Med. 2014;46(2):180-3.

24. Murphy D et al. Myocarditis associated with Campylobacter jejuni. N Z Med J. 2013:126(1375):95-8

25. Hull SR, Varma MP. Myopericarditis following Campylobacter infection. Ir J Med Sci. 2011:180(3):753-5.

26. Kratzer $\mathrm{C}$ et al. Acute cardiac disease in a young patient with Campylobacter jejuni infection: a case report. Wien Klin Wochenschr. 2009;122(9-10):315-9.

27. Heinzl $B$ et al. Campylobacter jejuni infection associated with myopericarditis in adolescents: report of two cases. Eur J Pediatr. 2009:169(1):63-5.

28. Turpie DF et al. Food-the way to a man's heart: a mini-case series of Campylobacter perimyocarditis. Scand J Infect Dis. 2009;41(6-7):528-31.

29. Turley AJ, Crilley JG, Hall JA. Acute myocarditis secondary to Campylobacter jejuni enterocolitis. Resuscitation. 2008:79(1):165-7.

30. Braun KP et al. [Campylobacter-jejuni-associated hepatitis and myocardial injury]. Med Klin (Munich). 2008;103(5):346-8.

31. Becker $\mathbf{S}$ et al. Is the incidence of perimyocarditis increased following Campylobacter jejuni infection? Eur J Clin Microbiol Infect Dis. 2007:26(12):927-9.

32. Mera V, Lopez T, Serralta J. Take traveller's diarrhoea to heart. Travel Med Infect Dis. 2007;5(3):202-3.

33. Kotilainen $\mathrm{P}$, Lehtopolku M, Hakanen AJ. Myopericarditis in a patient with Campylobacter enteritis: a case report and literature review. Scand J Infect Dis. 2006:38(6-7):549-52

34. Hannu T et al. Three cases of cardiac complications associated with Campylobacter jejuni infection and review of the literature. Eur J Clin Microbiol Infect Dis. 2005;24(9):619-22.

35. Reda E, Mansell C. Myocarditis in a patient with Campylobacter infection. N Z Med J. 2005;118(1221):U1634

36. Hamdulay SS et al. A diarrhoeal illness complicated by heart failure. Hosp Med. 2004;65(12):756-7.

37. Westling K, Evengard B. Myocarditis associated with Campylobacter infection. Scand J Infect Dis. 2001:33(11):877-8.

38. Wanby $P$, Olsen B. Myocarditis in a patient with salmonella and campylobacter enteritis. Scand J Infect Dis. 2001;33(11):860-2.

39. Sauvant $\mathrm{G}$ et al. [Diagnosis and course of myocarditis: a survey in the medical clinics of Zurich University Hospital 1980 to 1998]. Schweiz Med Wochenschr. 2000;130(36):1265-71.

40. Douchet MP et al. [Myopericarditis caused by Campylobacter jejuni]. Presse Med. 1999;28(23):1237

41. Williams A. First the chicken, then the egg; the heartburn came later. Med Health R I. 1997;80(5):163-5.

42. Sanchez JF et al. Campylobacter jejuni diarrhea and myopericarditis. An Med Interna. 1992;9(2):102-3.

43. Melby K, von der Lippe E, Bruun JN. Myocarditis and pericarditis in connection with Campylobacter jejuni infection. Tidsskr Nor Laegeforen. 1992;112(4):475-6. 
44. Florkowski CM et al. Campylobacter jejuni myocarditis. Clin Cardiol. 1984;7(10):558-9.

45. Ponka A et al. Carditis and arthritis associated with Campylobacter jejuni infection. Acta Med Scand. 1980;208(6):495-6.

46. Rahman M. Bacteraemia and pericarditis from Campylobacter infection. Br J Clin Pract. 1979;33(11-12):331. 4.

47. Lieber IH, Rensimer ER, Ericsson CD. Campylobacter pericarditis in hypothyroidism. Am Heart J. 1981;102(3 Pt 1):462-3.

48. Nowakowski M, Listopadzki D, Freeman S. Pericarditis associated with Campylobacter jejuni Enterocolitis, Hospital Physician; 2004.vol. 40. p. 39-42.

49. Lai T, Yadav R, Schrale R. Mimicking myocardial infarction: localized STsegment elevation in Campylobacter jejuni myopericarditis. Intern Med J. 2009;39(6):422-3.

Submit your next manuscript to BioMed Central and we will help you at every step:

- We accept pre-submission inquiries

- Our selector tool helps you to find the most relevant journal

- We provide round the clock customer support

- Convenient online submission

- Thorough peer review

- Inclusion in PubMed and all major indexing services

- Maximum visibility for your research

Submit your manuscript at www.biomedcentral.com/submit 